\title{
Chronotopes en MOUVEMENT. LES DIORAMAS CINÉMATOGRAPHIQUES DE DOMINIQUE GONZALEZ-FOERSTER
}

\begin{abstract}
Alexander Streitberger ${ }^{1}$
Cet article s'intéresse aux dioramas artistiques qui évoquent l'apparence, l'usage et la fonction du diorama historique tout en transformant ce système de présentation en dispositif cinématographique. Plus précisément, il sera question des dioramas de Dominique Gonzalez-Foerster où plusieurs couches temporelles et narratives se superposent et s'enchevêtrent pour créer un récit plurivoque proche du montage cinématographique. Le concept de chronotope de Bakhtine servira à comprendre la complexité temporelle et narrative de ces nouveaux dioramas cinématographiques.
\end{abstract}

La résurgence du diorama en tant que dispositif d'exposition artistique est un phénomène assez récent. Depuis les années 1990, on peut observer de nombreux artistes tels que Mark Dion, Damien Hirst, Hiroshi Sugimoto et Dominique GonzalezFoerster s'intéresser au diorama historique, issu du XIX $\mathrm{X}^{\mathrm{e}}$ siècle, tantôt pour ses qualités de présentation illusionniste tantôt pour sa fonction d'outil scientifique et didactique au sein des musées des sciences naturelles ${ }^{2}$.

1 Alexander Streitberger est professeur à l'Université catholique de Louvain.

2 Récemment, la place du diorama dans l'art contemporain a été abordée en lien avec le medium photographique (Bantleon \&Tragatschnig, 2013) et la technique de la taxidermie (Turner, 2013 ; Lange-Berndt, 2009).

Recherches en communication, $\mathrm{n}^{\circ} 46$ - Article publié le 24/04/2018 
Pour citer un exemple récent, la fondation d'entreprise Ricard accueillait, du 15 novembre 2016 au 7 janvier 2017, l'exposition Dioramas de l'artiste français Laurent Montaron (Lavrador, 2016). Enfermant des œuvres récentes de l'artiste dans un espace clos au moyen d'un dispositif de vitres, cette exposition était, en effet, conçue tel un diorama. Plus précisément, l'espace d'exposition et l'espace des œuvres étaient à la fois superposés et dissociés, car le spectateur, mis à distance par la vitre, percevait les œuvres exposées comme s'il s'agissait d'éléments d'une composition. Le spectateur se trouvait donc face à un dispositif aménagé à la manière d'une exposition, mais présenté comme une œuvre solidaire et unique. Au-delà de cette transformation d'un assemblage d'objets en une expérience purement visuelle, effectuée à travers la vitre, la question du diorama n'était cependant pas davantage développée, ni au niveau thématique ni par rapport à la fonction ou au contexte historique.

L'exposition Dioramas, organisée au Palais de Tokyo à Paris du 14 juin au 10 septembre 2017, constitue une autre preuve de l'actualité du sujet. Se vantant d'être «la première étude d'envergure sur le sujet ayant pour ambition de réconcilier les diverses ascendances du diorama », cette exposition se donne pour but de retracer « l'histoire de ses premières manifestations (ou "proto-dioramas") dans l'art religieux jusqu'à ses appropriations par les artistes contemporains » (Dohm, K., Garnier, C., Le Bon, L., \& Ostende, F., 2017, p. 9). De l'assemblage tridimensionnel Étant Donnés, conçu par Marcel Duchamp entre 1949 et 1966 comme véritable " machine à désir sexuel masculin » (Molderings, 2012, p. 74), à l'installation Hunting \& Collecting de 2016, créée par Sammy Baloji afin de jeter un regard critique vis-à-vis du colonialisme, l'exposition propose de situer de nombreuses installations d'art contemporain dans l'histoire du diorama « en tant qu'installation de matériaux et mise en espace de narrations » (Lange-Berndt, 2017, p. 286). Malgré l'intégration dans le parcours de l'exposition de quelques extraits de films tels que La Planète des singes (Franklin J. Schaffner, 1968) et La Nuit au musée (Shawn Levy, 2006), tous les deux témoignant de l'intérêt que le cinéma porte à cette autre machine illusionniste, 
et malgré les références évidentes au cinéma faites dans certaines œuvres de l'exposition, notamment The Theater Palace (Charles Matton,1989) et La Town (Cao Fei, 2014), force est de constater que cette dimension cinématographique n'est jamais abordée de manière explicite.

Le but de ce qui suit est donc de s'interroger sur la façon dont certaines œuvres d'art contemporain évoquent l'apparence, l'usage et la fonction du diorama historique tout en transformant ce système de présentation en dispositif cinématographique compris comme une machine narrative, poétique et critique à la fois. Pour ce faire, une étude de cas des dioramas de l'artiste française Dominique Gonzalez-Foerster semble particulièrement pertinente du fait que plusieurs couches temporelles et narratives s'y superposent et s'enchevêtrent pour créer un récit plurivoque proche du montage cinématographique. Basés sur le concept de chronotope de Mikhaïl Bakhtine, les trois dioramas que GonzalezFoerster a réalisés en 2010 dans le cadre de son exposition Chronotopes \& Dioramas à la Dia Art Foundation, New York constituent des capsules spatio-temporelles complexes qui, insérées dans un parcours d'exposition, tissent des liens multiples avec d'autres espaces et récits afin de créer " une sorte de situation magique » où l'illusion cinématographique du dispositif dioramique et l'expérience du spectateur se transforment en « deux espaces qui se regardent » (Gonzalez-Foerster, 2017, p. 272).

Avant de me pencher sur les dioramas cinématographiques de Gonzalez-Foerster, il me semble opportun de rappeler brièvement le contexte du diorama historique du $\mathrm{XIX}^{\mathrm{e}}$ siècle pour mieux comprendre le caractère cinématographique que ce dispositif illusionniste revêtait à l'origine avant d'être associé davantage au médium photographique. L'exemple du blockbuster The Lone Ranger, réalisé par Gore Verbinski en 2013, servira ensuite d'exemple pour montrer comment le diorama permet au cinéma hollywoodien de multiplier les couches narratives brouillant ainsi les limites entre la réalité et la fiction. Finalement, l'analyse des dioramas de Gonzalez-Foerster et de la manière dont l'artiste les a intégrés dans l'exposition présentée en 2015/2016 au 
Centre Pompidou à Paris révélera le caractère participatif de ce genre de dispositif dioramique. Le diorama cinématographique de Gonzalez-Foerster, telle est l'hypothèse, transforme le "spectateur désœuvré $»^{3}$ du cinéma (et du diorama historique), séparé de l'action par une surface faisant écran (l'écran de cinéma ou la vitre de diorama), en spectateur « passager, usager » invité à « organiser sa propre histoire en réponse à celle qu'il vient de voir, avec ses propres références » (Gonzalez-Foerster, 2015, p. 200).

\section{Le diorama dans l'histoire}

Du point de vue historique, le terme de diorama se réfère à deux dispositifs optiques bien distincts. Présenté en 1822 par Louis Daguerre et son associé Charles-Marie Bouton, le diorama est d'abord un dispositif illusionniste constitué d'énormes toiles translucides, peintes en trompe-l'œil et animées par des effets d'éclairage variés imitant le déplacement de personnes ou les changements d'éclairage que la journée apporte. Alors que les visiteurs de l'époque, habitués à l'expérience immersive du panorama en tant que « simulateur optique » (Oettermann, 1980, p. 12) insistaient encore davantage sur l'effet de réel de cet

3 J'emprunte ce terme à Raymond Bellour qui, dans son essai emblématique de 1984 « Le penseur pensif », définit le spectateur du cinéma comme un spectateur « désœuvré » ou " pressé », totalement pris par le déroulement de l'histoire sans prendre du recul sur ce qu'il regarde (les images) ou sur la situation dans laquelle il se trouve (la salle de cinéma). Le diorama fonctionne de manière similaire en créant l'illusion d'une scène réelle derrière laquelle le caractère artificiel et construit du dispositif disparaît. Cela ne signifie cependant pas que le spectateur du cinéma est d'office un spectateur passif. Toujours selon Bellour, certains effets ont le pouvoir d'interrompre le flux des images et de transformer le « spectateur pressé » en « spectateur pensif ». La photographie, en particulier, permet d'arrêter le film et de soustraire le spectateur à la fiction du cinéma : «Créant une distance, un autre temps, la photo me permet de penser au cinéma » (Bellour, 2002/1984, p. 77). Ceci dit, force est de constater que le dispositif du cinéma (et celui du diorama), contrairement aux installations dioramiques de Gonzalez-Foerster, impose au spectateur un point de vue immobile (ou quasi immobile pour le diorama) pour assurer une perception totale et continue. 
« environnement $[. .$.$] qui vise à captiver l'attention, à provoquer$ des perceptions visuelles aiguës, à transporter le spectateur au-delà de son propre corps »(Le Gall, 2013,p. 8), Walter Benjamin, ayant vécu l'émergence du cinéma comme spectacle d'images animées, y voit lui avant tout, un jalon important dans la préhistoire du médium cinématographique : « Il y a une sorte de précurseur espiègle de l'accéléré au cinéma, une accélération spirituelle, quelque peu malicieuse, du déroulement du temps [...]» (Benjamin, 1989/1982, p. 544).

Nous verrons que cette dimension cinématographique du diorama de Daguerre émerge à nouveau dans les dioramas artistiques qui seront abordés par la suite. En tant que dispositif d'exposition, ces derniers citent cependant un autre type de diorama ; celui du musée d'histoire naturelle situé au croisement entre art, science, pédagogie et culture du divertissement. Introduit vers la fin du XIX ${ }^{\mathrm{e}}$ siècle, le diorama des musées d'histoire naturelle est le fruit d'un nouveau rapport vis-à-vis de la nature, dans le cadre duquel on passe du système taxinomique de Linné à une approche biologique s'intéressant davantage à l'environnement et aux comportements des animaux (Kretschmann, 2006, p. 76 ; Köstering, 2003, p. 3). Composés d'une peinture panoramique de fond, de plantes réelles ou artificielles et de spécimens naturalisés, le diorama était censé présenter les animaux dans leur biotope, dans une situation spécifique, comme si le temps s'était arrêté à un moment précis. Pour cette raison, et malgré sa nature polymorphe et tridimensionnelle, le diorama a souvent été comparé à la photographie qui, selon André Bazin, « embaume le temps » (Bazin, 1958, p. 16).

Si Frank M. Chapman, conservateur au début du $\mathrm{XX}^{\mathrm{e}}$ siècle à l'American Museum of Natural History of New York, décrit le diorama comme une fenêtre donnant vue sur la nature même, c'est que cela correspond parfaitement à l'idée que l'on se faisait à l'époque de l'image photographique en tant que représentation précise et objective de la réalité. ${ }^{4}$ Dans le Cobb's Island Group,

4 Chapman utilise cette métaphore de la fenêtre ouverte sur le monde, empruntée au traité De Pictura de Leon Battista Alberti, dans un article publié en 1909 dans un guide du American Museum of Natural History : 
exécuté en 1903 par Chapman et constituant un des premiers habitat dioramas aux États-Unis, on retrouve un paysage peint, des oiseaux naturalisés et des objets naturels et artificiels étant arrangés de la sorte que le spectateur eût l'illusion d'observer une scène réelle, arrêtée dans le temps, afin qu'il puisse étudier les animaux dans leur environnement naturel (Minichiello \& White, 1997, p. 393).

Plus récemment, cette analogie entre diorama et photographie a été soulignée par Bodo von Brauchitsch selon lequel les photographies que Henning Bock a réalisées des dioramas de l'American Museum of Natural History sont des « photographies de photographies », sous-entendant par-là que le diorama lui-même fonctionne comme une image photographique (von Brauchitsch, s. d.). Citons encore Michelle Henning qui compare le caractère indiciel de la photographie, c'est-à-dire le rapport causal entre l'image et l'objet qu'elle représente, au procédé de la taxidermie appliqué aux animaux placés dans les dioramas, selon lequel la peau est posée par-dessus une structure modelée reconstituant les formes de l'animal (Henning, 2006 ; Henning, 2007).

\section{Une machine à voyager dans le temps}

La définition du diorama considéré tel un dispositif photographique fait oublier qu'il était, dès le début, une construction hybride et intermédiale dans laquelle se croisent les matériaux, les médias et les disciplines les plus divers, tels que la peinture, la sculpture, la photographie, le panorama, les sciences naturelles ou encore l'optique. C'est précisément cette ambivalence entre l'effet illusionniste du diorama et son hétérogénéité matérielle et culturelle qui semble avoir attiré l'intérêt des artistes ces dernières décennies. Alors que la fonction du diorama historique était de dissimuler cette hétérogénéité en faveur de l'illusion d'un espace naturel, cohérent et unitaire,

« one seems to be looking through a window on nature itself » Frank M. Chapman, The Habitat Groups of North American Birds, Guide Leaflet Series No. 28, AMNH (February 1909), p. 166. Cité dans : Wonders, p. 205 ; voir aussi : Quinn, 2006, p. 149. 
certains artistes contemporains, tels que Thomas Hirschhorn, Mark Dion, et Dominique Gonzalez-Foerster, recourent à ce dispositif justement pour souligner son côté hybride qui leur permet de créer un contraste entre l'idée moderniste de l'art comme objet esthétique autonome et les concepts de groupe biologique et de milieu écologique tels qu'ils émergent au cours du XIX ${ }^{\mathrm{e}}$ dans le discours des sciences naturelles (Kretschmann, 2006, p. 3). Selon Mark Dion, les dioramas « représentent l'acceptation et l'intégration de l'évolution et de l'écologie dans les musées [...] Les dioramas fonctionnent comme des machines à voyager dans le temps, non seulement vers le lieu et la période qu'ils représentent, mais aussi vers le moment spécifique de leur construction »(Dion, 1997, p. 137) 5 .

En tant que machines à voyager dans le temps, ces dioramas sont mobiles, ils traversent l'espace et le temps pour s'ouvrir sur ce que Dion a défini, dans le même article, l'" espace cinématographique » (Dion, 1997, p. 137). C'est précisément ce caractère ou ce potentiel cinématographique que Dion attribue au diorama et dont il sera ici question.

Le potentiel narratif et cinématographique du diorama a été également découvert par la puissante machine à illusions qu'est le cinéma hollywoodien. Nombreux sont les films qui jouent sur les effets de présence et de dédoublement du réel que le dispositif du diorama provoque dans la perception du spectateur. Alors que $L a$ Planète des singes (Franklin J. Schaffner, 1968) invertit les rôles entre l'homme comme créateur du diorama et l'animal comme objet exposé lors d'une scène où le protagoniste s'intègre dans un groupe d'hommes naturalisés pour se cacher de ses persécuteurs singes, La Nuit au musée (Shawn Levy, 2006) estompe les limites entre réalité et fiction en rendant la vie aux figures figées dans les dioramas du American Museum of Natural History pour qu'elles envahissent l'espace réel du musée. Pour approfondir le rapport complexe entre représentation et présentation, entre réalité et

$5 \ll[\ldots]$ represent the acceptance and integration of evolution and ecology into the museum. [...] Dioramas function as time machines, not only to the place and period they represent but also to the particular moment of their construction ». Ma traduction. 
fiction, que le diorama permet au cinéma d'établir - on pourrait aussi parler d'une mise en abyme de la machine illusionniste du cinéma même - je me réfère par la suite au film The Lone Ranger, réalisé par Gore Verbinski, produit par Disney et sorti en salle en 2013. Le film raconte les aventures fantastiques de l'indien Tonto, interprété par Johnny Depp, et son partenaire, le Lone Ranger, interprété par Armie Hammer. Dans le générique de ce western, un garçon d'environ 12 ans entre dans une tente de foire foraine où il découvre divers dioramas d'animaux avant de s'arrêter devant une scène présentant l'indien Tonto, placé à côté de sa tente et entouré d'un paysage de western éblouissant. Dans la scène qui suit, ce diorama se révèle comme une véritable machine spatio-temporelle qui nous propulse dans l'histoire du film.

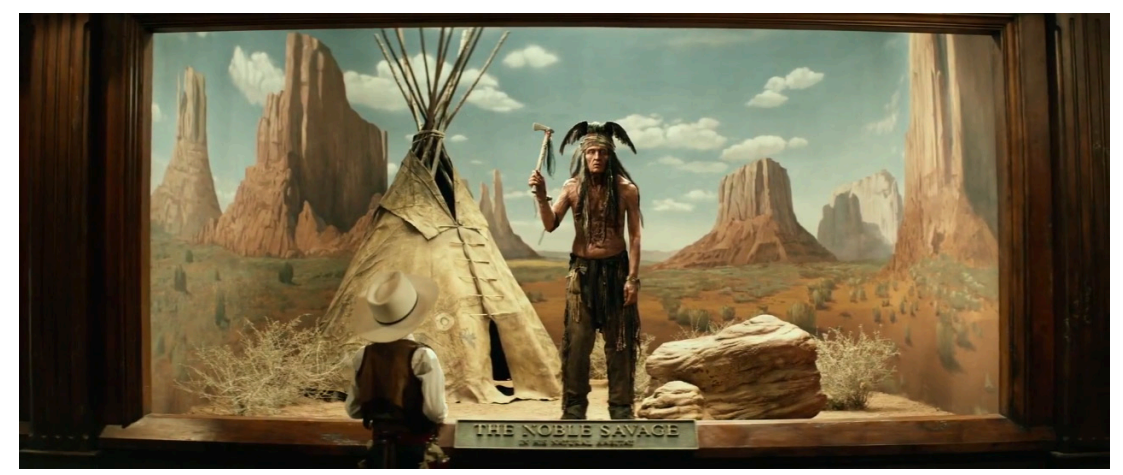

Figure 1. Gore Verbinski, The Lone Ranger, 2013

C'est face au diorama de l'indien que l'improbable se produit. Avec le clin d'œil que Tonto fait au garçon, le diorama prend vie, les frontières entre fiction et réalité sont brouillées, et l'histoire peut commencer. On voit les mains de Tonto traverser la vitre pour effectuer le troc de cacahuètes contre un rat mort. De toute évidence, cet échange à travers l'espace fictif de la représentation et l'espace réel de la tente est une mise en abyme de la situation du cinéma dans laquelle l'écran fonctionne comme surface de démarcation entre la salle de l'auditoire et la narration du film. Et pourtant, le diorama de Tonto ne marque pas uniquement la limite entre la réalité et la fiction. L'analogie entre la peinture 
panoramique du fond et le paysage de la première rencontre entre les deux héros dans l'histoire racontée par la suite nous indique que le diorama occupe une fonction centrale pour la narration filmique. À la fois image-souvenir, embaumant littéralement un moment du passé, et déclencheur du récit de Tonto présentant les événements comme un tourbillon d'actions en cascade aussi absurdes qu'acrobatiques, le diorama constitue une véritable machine narrative qui absorbe et relance l'histoire du Lone Ranger.

Ce va-et-vient entre passé et présent, ou encore entre image et réalité, s'exprime pleinement dans la dernière scène du générique lorsque l'action des deux héros, en plein cambriolage de banque, est gelée au moment de leur saut vers l'avant. Alors que l'arrêt sur image signifie le temps révolu de la légende, le mouvement de la tête de Tonto à l'intérieur de l'image figée raccroche au hic et nunc de la tente, ce geste étant provoqué par une question posée par le garçon; question qui ramène Tonto du terrain de la légende à la réalité triviale de la fête foraine.

On l'aura compris, dans The Lone Ranger le diorama n'apparaît pas comme une fenêtre prétendant offrir un regard objectif et scientifique sur l'environnement naturel des spécimens présentés. Il se présente plutôt comme un espace d'histoire en devenir, un champ fictionnel ou imaginaire dont le but n'est pas uniquement de restituer le passé, mais également de générer des images et des histoires au sein du film lui-même.

\section{Chronotopes et exposition cinématographique}

Les dioramas poétiques de Dominique GonzalezFoerster représentent une autre forme de machines narratives cinématographiques. Dans le cadre de son exposition individuelle Chronotopes \& Dioramas, organisée au sein de la Dia Art Foundation en 2010, l'artiste aménageait trois dioramas dans une annexe de 1'Hispanic Society of America transformée en espace d'exposition (Gonzalez-Foerster, 2010). Ces trois dioramas, au lieu de présenter des animaux dans leurs environnements naturels, mettaient plutôt en scène des livres qui, selon l'artiste, étaient 
censés entretenir un rapport associatif avec les paysages qui les accueillaient : les tropiques, le désert et l'Atlantique. Dominique Gonzalez-Foerster a choisi ces trois biosphères pour avoir, en tant qu'étrangère, un premier accès à l'Amérique, et c'est à travers ces livres que ce rapport topographique est articulé. L'artiste les a sélectionnés pour réagir à un manque qu'elle avait pu constater lorsqu'elle fouillait la bibliothèque de la société espagnole dont la richesse en livres anciens contrastait de manière frappante avec le nombre très réduit d'ouvrages du $\mathrm{XX}^{\mathrm{e}}$ siècle. L'idée à l'origine des dioramas était donc de compléter, en partie, la collection de livres existante en l'extrapolant dans le présent (GonzalezFoerster, 2010, p. 49).

En guise d'illustration, le diorama du désert abrite des livres de Roberto Bolaño, Jorge Luis Borges, Ray Bradbury, Carlos Castaneda, John Fante, Frank Herbert, Dorothy M. Johnson et Dorothy Scarborough. Dispersés à travers un paysage aride et désolé, ces ouvrages sont présentés comme des organismes vivants, dont la survie est menacée, à l'image des animaux naturalisés des dioramas historiques dont l'un des objectifs principaux était justement la protection de la faune et la flore (Wonders, 1993, p. 161). Or, les dioramas sont des espaces bioesthétiques hermétiquement clos. Il est impossible de pénétrer la vitre qui sépare l'intérieur de l'extérieur et empêche ainsi la lecture des livres. Il s'agit donc de capsules spatio-temporelles dont les habitants, les livres, racontent des histoires de lieux, de temps et de cultures variés sans être accessibles aux visiteurs de l'exposition qui se rendent compte de leur statut de spectateurs privés du plaisir de lecture. Il ne s'agit cependant pas de caisses isolées, de pures simulations d'un monde imaginaire. 


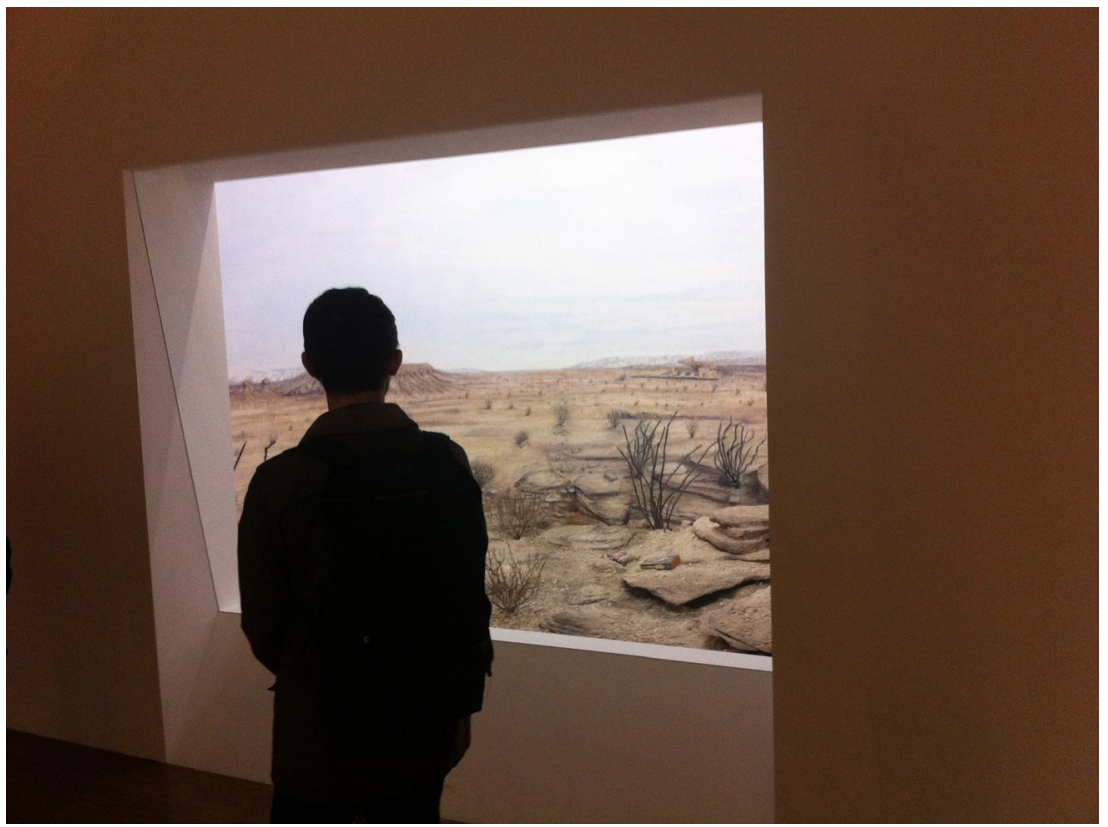

Figure 2. Dominique Gonzalez-Foerster, Chronotopes \& Dioramas (Desertic), vue de l'exposition au Centre Pompidou, Paris, 2009/2015. Image : Alexander Streitberger

En tant que dispositifs-œuvres, les dioramas s'inscrivaient en effet dans un dispositif-installation plus large qui est celui de l'exposition. Franchissant la porte d'entrée de l'espace d'exposition, le visiteur se trouvait devant une cloison qui le séparait des dioramas et qui le confrontait avec un panorama monumental composé de citations extraites des livres ainsi cloîtrés. Présenté sous forme de lignes arquées, de mots isolés et de blocs verticaux, ce collage vaste prenait la forme d'un paysage, invitant le visiteur à entrer dans l'espace imaginaire des dioramas qu'il allait découvrir par la suite de l'autre côté (GonzalezFoerster, 2010, p. 51). Ce type de poésie visuelle est inspiré, de toute évidence, du poème Un coup de dés jamais n'abolira le hasard dans lequel Stéphane Mallarmé bouleversait la linéarité du texte en faveur d'une «mobilité de l'écrit » censée libérer les mots de leurs carcans syntagmatique et sémantique (Mallarmé, 1998/1897, p. 391). 
Il en ressort que les dioramas font partie d'une mise en scène complexe où les dispositifs de présentation, de savoir et de conservation du panorama, du diorama et de la bibliothèque sont confrontés avec les stratégies avant-gardistes du collage et de la poésie visuelle afin de soulever la question du livre en tant qu'espèce menacée d'extinction. Comme le souligne Guillaume Le Gall, les dioramas de Gonzalez-Foerster imitent un dispositif optique issu du $\mathrm{XIX}^{\mathrm{e}}$ siècle tout en désignant " une catégorie littéraire de la forme et du contenu » dans le sens de Bakhtine (Le Gall, 2015, p. 21).

Un autre aspect fondamental est la question de l'exposition. " Contrairement à l'œuvre autonome », explique GonzalezFoerster, « toute exposition requiert un contexte. C'est un médium contextuel. Pour moi, le terme de contexte se charge aujourd'hui d'une dimension plus historique. Je le vois davantage comme une question de temps que comme une question d'espace " (Gonzalez-Foerster, 2010, 50-51). Ce qui explique le choix du terme chronotopes en guise de titre de l'exposition. Proposée par le théoricien de la littérature Mikhaïl Bakhtine, la notion se réfère à la spatialisation du temps dans un récit fictionnel ou non :

Dans le chronotope de l'art littéraire a lieu la fusion des indices spatiaux et temporels en un tout intelligible et concret. Ici, le temps se condense, devient compact, visible pour l'art, tandis que l'espace s'intensifie, s'engouffre dans le mouvement du temps, du sujet, de l'Histoire. Les indices du temps se découvrent dans l'espace, et ce dernier est perçu et mesuré d'après le temps (Bakhtine, 1978/1975, p. 237).

En effet, on retrouve aussi ce phénomène de condensation spatio-temporelle dans les expositions de Gonzalez-Foerster où des éléments provenant de différentes époques et de contextes divers se joignent et s'entrecroisent. L'exposition que l'artiste a présentée du 23 septembre 2015 au 1er février 2016 au Centre Pompidou à Paris nous catapulte, de surcroît, dans le futur. Intitulé 1887 - 2058 et faisant passer en revue les dernières trente années de son activité artistique, cette exposition n'était pas une 
rétrospective conventionnelle. Elle se présentait plutôt sous forme d'un labyrinthe constitué de chambres, d'environnements et de passages et organisé selon une chronologie ouverte s'étendant de 1887, l'année de la construction du palais de cristal à Madrid où l'artiste avait réalisé une installation en 2014, à l'année 2058, date qui se réfère à son exposition à la Tate Modern, Londres, organisée en 2008, dans laquelle l'artiste projetait la métropole britannique cinquante ans plus tard (Gonzalez-Foerster, 2015).

Plus précisément, Gonzalez-Foerster concevait l'exposition 1887 - 2058 comme un espace de réflexion, de déambulation et de dialogue, situé entre réalité et fiction, vécu et imagination, mêlant différentes époques et médiums pour créer un monde habité par ses idées, ses pensées, ses images, mais également par celles du spectateur que l'artiste conçoit comme un « spectateurlecteur » ou encore un "spectateur-détective » : à lui de trouver des indices pour ensuite « organiser sa propre histoire en réponse à celle qu'il vient de voir, avec ses propres références » (GonzalezFoerster, 2015, p. 200 ; Gonzales-Foerster, Huyghe \& Parreno, 1998, p. 82). Le critique d'art Eric Troncy décrit l'expérience d'une exposition de Gonzalez-Foerster à juste titre comme une expérience cinématographique. En effet, les œuvres, représentant chacune une capsule spatio-temporelle, sont disposées au long du parcours de l'exposition comme des microhistoires dont le montage final doit être réalisé par l'imagination du spectateur. Et Troncy d'écrire : « Le spectateur doit travailler. À lui de terminer les scénarios, de procéder aux adaptations, de faire le montage de cette exposition tel un film» (Troncy, 1998, p. 48). Les espaces de l'exposition sont donc conçus comme un scénario de film dont les protagonistes sont issus de grandes œuvres de la littérature, du cinéma et du théâtre pour être réintégrés dans l'exposition organisée selon des chambres dont chacune a une atmosphère spécifique et raconte une histoire différente.

Dans RWF (chambre) de 1993, cette dimension cinématographique est doublement présente. Aménagée d'après la description que Robert Katz donne de la chambre de Rainer Werner Fassbinder dans sa biographie L'amour est plus froid que la mort : une vie de Rainer Werner Fassbinder (Katz, 1988), 
cette pièce n'est pas uniquement la chambre du grand cinéaste allemand, ou son habitat, pour rester dans la terminologie du diorama ; elle est également le produit d'un récit biographique, dans lequel elle est décrite comme « une grotte de grand luxe » ou encore comme « une tombe » (Katz, 1988, p. 170) tout en étant issue de l'imagination de l'artiste qui transforme le récit littéraire en ambiance environnementale :

La chambre, c'est pour moi une dimension naturelle de l'art, le premier lieu où l'on accroche des choses personnelles ou collectives. C'est un espace mental où l'on compose une ambiance. [...] Mes chambres sont comme des images, mais dans lesquelles on peut rentrer. On est physiquement entouré par l'image, un peu comme au cinéma. D'ailleurs, il y a chez moi l'obsession d'un récit, d'une narration, fûtelle spatiale (Gonzalez-Foerster, 2015, p. 190 ; Colard, 1998, p. 67).

Autrement dit, les chambres de Gonzalez-Foerster sont des environnements qu'il faut expérimenter non seulement dans l'espace, mais aussi dans le temps. De là leur qualité cinématographique.

Par ailleurs, au centre de ce labyrinthe de chambres et d'environnements, se trouvait le diorama du dessert situé visà-vis du textorama, les deux constituant un environnement dans lequel le visiteur était tiraillé entre ces deux dispositifs de représentation si différents ; l'un plat, fragmenté et textuel, l'autre profond, homogène, et illusionniste. La date inscrite en bas de la cloison perpendiculaire au mur du diorama ne laisse aucun doute sur la place centrale que cet espace dio-texto-ramique occupait dans l'exposition : c'est l'année 2058 qui referme la parenthèse chronologique de l'exposition dans un futur encore à inventer.

On l'aura compris, le diorama est au cœur d'une machine narrative susceptible de " fusionner fait et fiction, illusion et narration, de la sorte que sont conjurées le passé et le futur à la 
fois $»^{6}$ (Cooke, 2010, p. 51). Déjà en 1998, Gonzalez-Foerster avouait :

Je passe plus de temps «à l'intérieur » des films que dans les expositions, c'est forcement contaminant à force. Cela permet aussi d'aborder l'exposition d'une tout autre façon. En fait, je crois de plus en plus à une histoire secrète de l'exposition à travers le cinéma... (Gonzalez-Foerster, 2015, p. 193 ; Troncy, 1998, p. 50).

Le diorama, semble-t-il, constitue le point d'orgue provisoire de cette histoire secrète.

L'exemple du blockbuster The Lone Ranger, avec lequel j'ai commencé mes réflexions, fournit un autre indice du potentiel cinématographique du diorama. Ainsi le diorama comme dispositif scientifique et pédagogique de la modernité, conçu comme une fenêtre prétendant offrir une vue objective et neutre sur un pan de nature intacte, se transforme en un environnement narratif qui s'inscrit dans un contexte plus large, celui du cinéma ou de l'exposition, pour s'ouvrir sur un autre monde qui est celui de la fiction et de l'imagination. Or, les espaces dioramiques du musée d'histoire naturelle et du film hollywoodien impliquent tous les deux l'attitude d'un " spectateur désœuvré » qui se trouve face à un dispositif immersif, mais impénétrable. Avec les assemblages tridimensionnels de Gonzalez-Foerster, le diorama s'ouvre vers d'autres espaces de présentation et de représentation - le panorama, le texte, la chambre, l'exposition - tout en impliquant le spectateur dans un jeu dialogique entre inclusion (dans l'environnement dio-texto-ramique) et exclusion (du monde présenté à l'intérieur du diorama), entre espace fictionnel (de l'image) et espace réel (de l'exposition), ou encore entre le temps passé (du diorama historique) et le futur dans lequel le spectateur est invité à projeter les expériences qu'il fait dans le présent lors de sa visite de l'exposition.

6 « In melting fact and fiction, illusion and narrative, in ways that conjure the future as well as the past [...]. » Ma traduction. 


\section{Références}

Bakthine, M. (1978/1975). Esthétique et théorie du roman. Paris : Gallimard.

Bantleon, K., \& Tragatschnig, U. (2013). Willful Deceptions: Aesthetic Illusion at the Interface of Painting, Photography and Digital Images. Dans W. Wolf, W. Bernhart \& A. Mahler (Éd.), Immersion and Distance: Aesthetic Illusion in Literature and Other Media (pp. 263-293). Amsterdam, New York : Rodopi.

Bazin, A. (1958). Qu'est-ce le cinéma? Paris : Les éditions du CERF.

Bellour, R. (2002/1984). Le spectateur pensif. Dans R. Bellour, L'Entre-Images. Photo. Cinéma. Vidéo (pp. 75-84). Paris : Éditions de la Différence.

Benjamin, W. (1989/1982). Paris. Capitale du XIXe siècle. Le livre des passages, sous la direction de Rolf Tiedemann, trad. par Jean Lacoste. Paris : Les éditions du CERF.

Brauchitsch, B. (s. d.). Animals and Landscapes, Lumas. Disponible à : http://be-fr. lumas.com/artist/henning_bock/.

Colard, J.-M. (1998). Chambres à part, Les Inrockuptibles, 153, p. 67.

Cooke, L. (2010). Frameworks. Dans D. Gonzalez-Foerster, Chronotopes \& Dioramas, cat., Dia Art Foundation New York (pp. 47-59). New York : Dia Art Foundation.

Dion, M. (1997). The Natural History Box : Preservation, Categorization and Display (1995). Dans L. Graziose Corrin, M. Kwon, \& N. Bryson (Éd.), Mark Dion (pp. 134139). London : Phaidon.

Dohm, K., Garnier, C., Le Bon, L., \& Ostende, F. (2017). Dioramas, cat., Palais de Tokyo (Paris), Schirn Kunsthalle Frankfurt (Francfort-sur-le-Main), Paris : Flammarion.

Gonzalez-Foerster, D., Huyghe, P., \& Parreno, P. (1998). Dominique Gonzalez-Foerster,

Pierre Huyghe et Philippe Parreno, cat., ARC/Musée d'art moderne de la Ville de Paris, 30 oct. 1998 - 10 janv. 1999, Paris : Paris musées.

Gonzalez-Foerster, D. (2010). Chronotopes \& Dioramas, cat., Dia Art Foundation New York. New York : Dia Art Foundation.

Gonzalez-Foerster, D. (2015). Dominique Gonzalez-Foerster. 1887 - 2058, cat., Centre Pompidou Paris, 23 oct. 2015 - 1er févr. 2016, Paris : Editions du Centre Pompidou.

Gonzalez-Foerster, D. (2017). Chronotopes et Dioramas. Dans K. Dohm, C. Garnier, L. Le Bon \& F. Ostende. Dioramas, cat., Palais de Tokyo (Paris), Schirn Kunsthalle Frankfurt (Francfort-sur-le-Main) (pp. 270-273). Paris : Flammarion.

Henning, M. (2006). Skins of the Real: Taxidermy and Photography. Dans B. Snaebjornsdottir \& M. Wilson (Éd.), Nanoq: flat out and bluesome. A Cultural Life of Polar Bears (pp. 136-147). London : black dog.

Henning, M. (2007). Anthropomorphic taxidermy and the death of nature: The curious art of Hermann Ploucquet, Walter Potter and Charles Waterton.Victorian Literature and Culture, 35 (2), pp. 663-678.

Katz, R. (1988). L'amour est plus froid que la mort : une vie de Rainer Werner Fassbinder. Paris : Presses de la Renaissance.

Köstering, S. (2003). Natur zum Anschauen. Das Naturkundemuseum des deutschen Kaiserreichs 1871-1914. Köln, Weimar, Wien : Böhlau.

Kretschmann, C. (2006). Räume öffnen sich. Naturhistorische Museen im Deutschland des 19. Jahrhunderts. Berlin : Akademieverlag.

Lange-Berndt, P. (2009). Animal Art: Präparierte Tiere in der Kunst 1850-2000. Munich : Metzel.

Lange-Berndt, P. (2017). « Diorama-Drama »! Le diorama dans l'art contemporain. Dans K. Dohm, C. Garnier, L. Le Bon \& F. Ostende. Dioramas, cat., Palais de Tokyo 
(Paris), Schirn Kunsthalle Frankfurt (Francfort-sur-le-Main) (pp. 286-288). Paris : Flammarion.

Lavrador, J. (2016). Laurent Montaron, emballage sous vitre, Libération, 4 décembre 2016. Disponible à : http://next.liberation.fr/arts/2016/12/04/laurentmontaron-emballage-sous-vitre_1532906?utm_campaign=Echobox\&utm medium=Social\&utm_source $=$ Twitter\#link_time $=1480931289$.

Le Gall, G. (2013). La peinture mécanique. Le diorama de Daguerre . Paris : Mare \& Martin.

Le Gall, G. (2015). Rémanences du diorama chez Dominique Gonzalez-Foerster, Espace, 109, pp. 18-27.

Mahler (Éd.), Immersion and Distance: Aesthetic Illusion in Literature and Other Media (pp. 263-293). Amsterdam, New York : Rodopi.

Mallarmé, S. (1998/1897). Euvres complètes, vol. 1, sous la direction de Bertrand Marchal. Paris : Gallimard.

Minichiello, J. K., \&White, A. W. (Éd.) (1997). From Blue Ridge to Barrier Islands: An Audubon Naturalist Reader. Baltimore: Johns Hopkins University Press.

Molderings, H. (2012). Die nackte Wahrheit. Zum Spätwerk von Marcel Duchamp, Munich : Carl Hanser.

Oettermann, S. (1980). Das Panorama. Die Geschichte eines Massenmediums. Frankfurt am Main : Syndikat.

Quinn, S. Ch. (2006). Windows on Nature. The Great Habitat Dioramas of the Amercian Museum of Natural History. New York: Abrams.

Troncy, E. (1998). Gonzalez-Foerster, Huyghe, Parreno : l'image en questions, Beaux Arts magazine, 174, pp. 46-51.

Turner, S. S. (2013). Relocating "Stuffed» Animals : Photographic Remediation of Natural History Taxidermy, Humanimalia, 4:2. Disponible à : http://www.depauw. edu/humanimalia/issue \%2008/turner.html.

Wonders, K. (1993). Habitat Dioramas: Illusions of Wilderness in Museums of Natural History. Uppsala : Almqvist \& Wiksell.

\section{@® $\Theta \Theta$}

Publié sous la licence Creative Commons

«Attribution - Pas d'Utilisation Commerciale - Pas de Modification 4.0 International» (CC BY-NC-ND) 